\title{
Práctica físico-deportiva, actividades de ocio y concepción sobre la Educación Física en escolares deA Conuña \\ Sports Practice, Leisure-time Activities, and Notion of Physical Education in Schoolchildren from A Coruña \\ *Ramón Chacón Cuberos, **Víctor Arufe Giráldez, *Tamara Espejo Garcés, ***Javier Cachón Zagalaz, *Félix Zurita Ortega, ***Denis Castro García \\ *Universidad de Granada (España), **Universidad de A Coruña (España), ***Universidad de Jaén (España)
}

Resumen. El presente estudio persigue como principales objetivos concretar los deportes más practicados en una muestra de escolares, así como relacionar el nivel de práctica físico-deportiva con el nivel de esfuerzo, el tipo de ocio y la concepción sobre la Educación Física (EF). Se realizó un estudio descriptivo de corte transversal en una muestra de 818 escolares de tercer ciclo de educación primaria de la provincia de A Coruña (España). La representación por sexo fue del 52\% para los niños y un 48\% para las niñas, con edades comprendidas entre los 10 y 13 años de edad ( $M=11,17$; DT $=0,44$ ). Como instrumento se emplea un cuestionario Ad Hoc para el registro de variables sociodemográficas y de tipo deportivo. Los resultados revelan que uno de cada diez escolares no practica ningún deporte, mientras que los deportes colectivos con contacto de forma federada son los más practicados. El fútbol y los deportes acuáticos fueron los deportes más populares, mientras que el balonmano y el atletismo eran los menos practicados. El tiempo invertido en la práctica físico-deportiva se relacionó de forma positiva con el ocio deportivo y la aptitud percibida para la EF, y de forma negativa con el ocio digital. El nivel de esfuerzo implicado en el deporte correlacionó negativamente con el ocio deportivo, la concepción sobre la EF y la aptitud percibida para la EF. Finalmente, el ocio deportivo reveló una relación negativa con el ocio digital y el ocio académico, así como una relación positiva con la concepción sobre la EF y la aptitud percibida para la AF.

Palabras clave. Deporte; Educación Física; Ocio; Escolares.

Abstract. This research aims to define the most popular sports in a sample of schoolchildren, and to relate sports practice levels with the level of effort implicated, the kind of leisure-time activities, and the notion about Physical Education (PE). A descriptive and cross-sectional study was conducted on a sample of 818 students of third cycle of Primary Education from the province of A Coruña (Spain). Gender representation was 52\% for boys and 48\% for girls, with age range between 10 and 13 years old $(M=11,17$; $S D=0,44)$. The instrument employed was an Ad-Hoc questionnaire for recording sociodemographic factors and sports-related variables. Results show that one out of ten schoolchildren does not practice any sport, whilst collective sports with contact, mainly played in a federated way, are the most practiced. Football and water sports were the most popular, whereas track and field and cycling were the least practiced. Furthermore, the time spent on sports practice was positively related with leisure-time sports and perceived aptitude towards PE, and negatively correlated with digital leisure time. The level of effort involved in sports was negatively correlated with sport leisure time, notion of PE, and perceived aptitude towards PE. Finally, sports leisure time was found to have a negative relation with academic and digital leisure times, as well as a positive relation with the notion of PE and perceived aptitude towards PE.

Keyword. Sport; Physical Education; Leisure Time; Schoolchildren.

\section{Introducción}

El continuo desarrollo tecnológico y los cambios producidos en los estilos de vida implicados en la sociedad actual ha generado niveles elevados de sedentarismo en un amplio rango de edades (Anderson et al., 2010; Chacón-Cuberos, Castro-Sánchez, Muros-Molina, EspejoGarcés, Zurita-Ortega y Linares-Manrique, 2016; Van Rooij, Schoenmakers, Vermulst, Vermulst, Van den Eijden y Van de Mheen, 2010). Esta problemática, unida al descenso de los niveles de práctica físico-deportiva que se produce desde edades tempranas (Alonso, Carranza, Rueda y Naranjo, 2014; Dias y Gama, 2016) y el empobrecimiento de la calidad de la dieta, configura estilos de vida poco saludables que se asocian a consecuencias negativas para la salud como son diabetes, hipertensión, obesidad o problemas músculo-esqueléticos (Estruch et al., 2013; OMS, 2015).

Autores como Arriscado, Muros, Zabala y Dalmau (2015) o Reverter, Plaza, Jové y Hernández (2014) ponen en relieve la importancia de desarrollar estrategias para la práctica de Actividad Física (AF) y deporte como medios para la disminución del exceso de peso y la mejora de enfermedades crónicas no transmisibles. De hecho, la práctica de AF y deporte ha aumentado considerablemente en las últimas décadas al constituir un medio de ocupación lúdico que genera efectos positivos a nivel fisiológico, cognitivo y socio-afectivo (Chacón, Espejo, Cabrera, Castro, López y Zurita, 2015; Reverter et al., 2014). Algunos de sus beneficios son la disminución del riesgo de padecer enfermedades cardiovasculares, el control de peso, tratamiento del estrés, la mejora de la autoestima o la socialización con pares, entre otros (Espejo, Cabrera, Castro, López, Zurita y Chacón, 2015; Moreno-Murcia, Borges, Mar-

Fecha recepción: 20-09-16. Fecha de aceptación: 06-02-17 Ramón Chacón Cuberos

ramonchaconcuberos@correo.ugr.es cos, Sierra y Huéscar, 2012).

En este sentido, resulta imprescindible conocer las principales motivaciones implicadas en la realización de actividad física y deporte (Isorna, Rial y Vaquero-Cristóbal, 2014; Rodríguez, 2016). De hecho, Owen, Astell,-Burt y Lonsdale (2013) sitúan la motivación como un factor indispensable en la consecución de un estilo de vida activo, la cual depende de aspectos individuales como creencias, cogniciones y valores, así como de influencias sociales como el apoyo familiar, los hábitos físico-deportivos del entorno inmediato o las interacciones de cooperación y competición con los pares (Kane, 2015; Méndez-Giménez, Fernández-Río, Cecchini y González, 2013), pudiendo actuar todos ellos como potenciadores o distractores. En este sentido, la Educación Física (EF), entendida como la área del currículo escolar que focaliza sus objetivos en el desarrollo de habilidades y competencias motrices, el desarrollo personal y la mejora de la calidad de vida (Kane, 2015; Navarro-Patón, Rodríguez y Eirín, 2016), constituye un instrumento esencial para motivar a los sectores más jóvenes de la población a la iniciación deportiva, generando actitudes que favorezcan un estilo de vida activo, saludable y prolongado en el tiempo(Espejo etal., 2015; MéndezGiménez et al., 2013; Reverter et al., 2014).

Ante esta perspectiva, diversos trabajos abordan la motivación hacia la práctica deportiva y la percepción sobre la importancia hacia la educación física desde la teoría de las metas de logro (García-Calvo, Sánchez, Leo, Sánchez y Amado, 2011; Isorna et al., 2014; MorenoMurcia, Zomeño, Marín, Ruiz y Cervelló, 2013). Por ejemplo, Barkoukis, Ntoumanis y Thogersen (2010) estudian las orientaciones de meta implicadas en EF, detectando como la disminución de las motivaciones orientadas a la tarea que se produce al aumentar la edad, genera mayor inactividad física. De este modo, resulta fundamental promover climas motivacionales que favorezcan una práctica hedonista y atractiva. En una línea similar, Moreno-Murcia et al. (2013) analizan las relaciones existentes entre el clima motivacional transmitido por el profesor del aula, diversos medidores psicológicos, la motivación 
autodeterminada y la importancia y utilidad que el escolar le concede a laEducación Física, concluyendo la importancia de generar motivación autodeterminada en el alumnado con el fin de promover su autonomía, competencia y relación con los demás.

Asimismo, y justificando la importancia de la inclusión delárea de EF en los planteamientos curriculares y en el diseño de programas de intervención, Martínez, Contreras, Aznar y Lera (2012) estudian los niveles de AF medidos con acelerómetro en una muestra de escolares, concluyendo que la AF de intensidad media y vigorosa realizada en la asignatura de EF suponía una porcentualidad importante del total realizado semanalmente. Esto revela el interés de promover motivaciones intrínsecas en la práctica deportiva desde las aulas, con el fin de que satisfagan necesidades psicológicas básicas y promuevan un estilo de vida activo (García-Calvo et al., 2011; Sicilia, Águila, González-Cutre y Moreno-Murcia, 2011).

Partiendo de esta motivación hacia la práctica de actividad física y deporte, Gómez-Mármol y De la Cruz-Sánchez (2013) ponen énfasis en la importancia de la percepción del alumnado sobre la aptitud en el área de EF, la cual se relaciona con la capacidad de hacer frente a las exigencias física y tareas, siendo fundamental el dominio de diversas competencias, una percepción como experiencia divertida y el desarrollo de motivaciones autodeterminadas. De hecho, Baena, Flores y Barbero (2007) estudian la implicación que las actividades físicas y deportivas ejercen en el currículo escolar de educación física, destacando la relevancia de que estas sean lúdicas, especializadoras, autóctonas, socializadoras, variadas y motivadoras.

Ante la importancia de la práctica físico-deportivo en edades tempranas para la promoción de la salud, este estudio persigue como principales objetivos:

- Concretar los principales deportes que realiza una muestra de escolares de Educación Primaria, así como el modo de práctica más común.

- Relacionar el nivel de práctica físico-deportiva con el nivel de agotamiento y esfuerzo implicado, el tipo de ocio que realizan los escolares y la concepción y aptitud percibida hacia la EF que poseen.

\section{Método}

\section{Diseño}

Se realizó un estudio no experimental, de tipo descriptivo y corte transversal. Asimismo, el diseño fue analítico y relacional, al concretar relaciones entre todas las variables.

\section{Participantes}

La muestra estuvo constituida por 818 estudiantes de Educación Primaria de la provincia de ACoruña, representados en un 52\% (n=425) por varones y en un $48 \%(n=393)$ por mujeres, con una edad comprendida entre los 10 y 13 años $(M=11,17 ; D T=0,44)$. La selección de los participantes se realizó a través de un muestreo aleatorio por conglomerados, considerando los escolares matriculados en tercer ciclo de Educación Primaria según centros públicos (59,7\%) y privados (40,3\%).

Tabla 1.

Características de la muestra

\begin{tabular}{|c|c|c|c|}
\hline & & $\mathbf{N}$ & $\%$ \\
\hline \multirow{2}{*}{ Sexo } & Varón & 425 & 52,0 \\
\hline & Mujer & 393 & 48,0 \\
\hline \multirow{2}{*}{ Tipología de centro } & Público & 488 & 59,7 \\
\hline & Privado & 330 & 40,3 \\
\hline
\end{tabular}

\section{Instrumentos y variables}

Se utilizó un cuestionario Ad Hoc, en el cual se incluyeron los aspectos sociodemográficos estudiados y aquellas variables relacionadas con la práctica físico-deportiva y actividades de ocio. Se consideraron las siguientes variables:

- Deportes: Se considera el deporte que practican los escolares, clasificados en 0 = Ninguno; 1 = Voleibol; 2 = Danza; 3 = Baloncesto; 4 = Fútbol; 5 = Deportes acuáticos - Natación, Vela, Waterpolo, etc.- ; 6 = Deportes de raqueta - Tenis, Pádel, Tenis de mesa, etc.- ; 7 = Balonmano; 8 = Patinaje; 9 = Atletismo; 10 = Deportes de combate -
Kárate, Judo, Boxeo, Taekwondo, etc.- ; 11 = Ciclismo; 12 = Gimnasia -Deportiva, Rítmica, etc.- ; 13 = Otros -Golf, Rugby, Equitación, Escalada, etc.- .

- $\quad$ Tipo de práctica: Se clasifica el deporte practicado en $1=$ No practica; 2 = Individual sin contacto; 3 = Individual con contacto; 4 = Colectivo sin contacto; 5 = Colectivo con contacto.

- Modo de práctica: Establece el modo en el que cada participante realiza la práctica deportiva, según 1 = No practica; 2 = Lúdica con pares; 3 =Actividad extraescolar; 4 =Actividad del ayuntamiento; 5 = Club federado.

- Práctica deportiva: Se considera el nivel de práctica deportiva realizada por los escolares de forma extraescolar según el número de horas semanales que emplean en su realización.

- Nivel de agotamiento: Especifica el nivel de agotamiento cuando se practica algún deporte. Se puntúa mediante una escala de tipo Likert de 5 opciones, donde 1 = Nada agotado y 5 = Muy agotado.

- Nivel de esfuerzo: Especifica el nivel de esfuerzo implicado en la práctica deportiva mediante una escala de tipo Likert de 5 opciones, donde 1 = Nada de esfuerzo y 5 = Mucho esfuerzo.

- Actividades de ocio: Comprende 4 categorías -Ocio digital, Ocio deportivo, Ocio con pares y Ocio académico-, las cuales son puntuadas mediante una escala de tipo Likert de 5 opciones, donde $1=$ Nada frecuente y 5 = Muy frecuente.

- Concepción sobre la EF: Define la concepción del alumno sobre la importancia de la Educación Física mediante una escala Likert de 5 opciones, donde 1 = Nada importante y 5 = Muy importante.

- Aptitud percibida sobre la EF: Concreta la aptitud que el alumno percibe de sí mismo para la Educación Física a través de una escala Likert de 5 opciones, donde 1 = Nada apto y 5 = Muy apto.

\section{Procedimiento}

En primer lugar se elaboró una carta informativa desde la Facultad de Ciencias de Educación de la Universidad de La Coruña con el fin de informar a los centros educativos de la naturaleza del estudio a realizar, así como solicitar la colaboración de los mismos. Posteriormente, los centros que aceptaron participar contactaron con los tutores legales de los escolares, con el fin de obtener su consentimiento informado. Transcurrido este proceso, se procedió con la aplicación de los instrumentos. Esta etapa se realizó en horario lectivo, con la presencia de los investigadores -previamente instruidos en dos reuniones-, y del tutor de cada grupo, con el fin de asegurar la correcta aplicación de los instrumentos. Se han cumplido las normas éticas de la Declaración de Helsinki de 1975 y se ha respetado el derecho de confidencialidad de los participantes. Asimismo, se ha informado a los centros educativos de los resultados obtenidos en la investigación.

\section{Análisis de los datos}

El análisis de los datos se ha realizado mediante el software estadístico IBM SPSS 22.0. Se emplearon frecuencias para los descriptivos básicos y correlaciones bivariadas para determinar las relaciones entre las variables. Se empleó la prueba del Chi-cuadrado de Pearson $(p>.05)$ para determinar la significatividad de las relaciones.

\section{Resultados}

La Tabla 2 muestra cuáles son los deportes más practicados por los escolares. El fútbol con un 30,4\% (n=249) representa el deporte de mayor popularidad, seguido de los deportes acuáticos -natación, waterpolo, vela, etc.- con un 13,0\% (n=106) y el baloncesto, representado en un $10,0 \%(n=82)$. El balonmano se constituyó como la discipli-

\begin{tabular}{lcclcc}
$\begin{array}{l}\text { Tabla } 2 . \\
\text { Deporte practicado }\end{array}$ & & & & & \\
\hline DEPORTE & $\mathrm{N}$ & $\%$ & DEPORTE & $\mathrm{N}$ & $\%$ \\
\hline Ninguno & 72 & 8,8 & Balonmano & 7 & 0,9 \\
Voleibol & 33 & 4,0 & Patinaje & 36 & 4,4 \\
Danza & 51 & 6,2 & Atletismo & 12 & 1,5 \\
Baloncesto & 82 & 10,0 & Dep. Combate & 47 & 5,7 \\
Fútbol & 249 & 30,4 & Ciclismo & 12 & 1,5 \\
Dep. Acuáticos & 106 & 13,0 & Gimnasia & 22 & 2,7 \\
Dep. Raqueta & 55 & 6,7 & Otros & 34 & 4,2 \\
\hline
\end{tabular}


Tabla 3.

\begin{tabular}{lccc}
$\begin{array}{l}\text { Tabla 3. } \\
\text { Práctica físico-deportiva }\end{array}$ & $\mathbf{N}$ & $\mathbf{\%}$ \\
\hline & No practica & 72 & 8,8 \\
& Individual sin contacto & 283 & 34,6 \\
Tipo de deporte & 52 & 6,4 \\
& Individual con contacto & 63 & 7,7 \\
& Colectivo sin contacto & 349 & 42,7 \\
\hline & Colectivo con contacto & 72 & 8,8 \\
& No practica & 133 & 16,3 \\
Modo de práctica & Lúdica con pares & 177 & 21,6 \\
& Actividad extraescolar & 83 & 10,1 \\
& Actividad del ayuntamiento & 353 & 43,2 \\
\hline
\end{tabular}

Tabla 4.

Coeficientes de correlación entre práctica físico-deportiva, agotamiento, nivel de esfuerzo, tipo de ocio y percepciones sobre la EF

\begin{tabular}{|c|c|c|c|c|c|c|c|c|}
\hline & AG & ESF & ODI & ODE & OP & OA & CE & $\mathbf{A E}$ \\
\hline$\overline{\mathbf{P D}}$ & $.326^{* *}$ & -.059 & $-.134^{* *}$ & $.245^{* *}$ & -.001 & -.017 & .015 & $.171^{* *}$ \\
\hline AG & & -.024 & $-.083^{*}$ & $.187^{* *}$ & -.029 & -.037 & .007 & .030 \\
\hline ESF & & & $.156^{* *}$ & $-.258^{* *}$ & .038 & -.063 & $-217^{* *}$ & $-.344^{-*}$ \\
\hline ODI & & & & $-324^{* *}$ & $.086^{*}$ & $-.139^{* *}$ & $-.084^{*}$ & $-.102^{+*}$ \\
\hline ODE & & & & & .039 & .059 & $.112^{* *}$ & $.214^{* *}$ \\
\hline OP & & & & & & .005 & .032 & .003 \\
\hline OA & & & & & & & .005 & -.062 \\
\hline
\end{tabular}

$\frac{\text { CE }}{{ }^{1} \text { PD: Práctica deportiva; AG: Agotamiento; ESF: Esfuerzo; ODI: Ocio Digital; ODE: Ocio }}$

Deportivo; OP: Ocio con Pares; OA: Ocio Académico; $C E$ : Concepción EF; AE: Aptitud EF.

$2 *$. La correlación es significante al nivel 0,05 (bilateral).

$3 * *$. La correlación es significativa al nivel 0,01 (bilateral).

na menos practicada con un $0,9 \%(n=7)$, seguido del atletismo y el ciclismo con unas porcentualidades del 1,5\% $(\mathrm{n}=12)$.

A continuación se revela el tipo de práctica físico-deportiva realizada así como el modo de práctica (Tabla 3). Se observa que un 8,8\% $(\mathrm{n}=72)$ de los participantes no realiza ningún deporte. Asimismo, un $42,7 \%$ ( $n=349)$ desempeña deportes colectivos con contacto y un $34,6 \%(n=283)$ individuales sin contacto, siendo las categorías con mayores porcentualidades. El modo de práctica denota la adhesión a clubes o práctica federada como el más común, adhiriéndose un 43,2\% ( $\mathrm{n}=353)$ de los participantes. Un 16,3\% ( $\mathrm{n}=133)$ practica deportes con sus pares de forma lúdica y un 21,6\% (n=177) lo hace en actividades extraescolares.

Finalmente se muestran los coeficientes de correlación entre la práctica deportiva, el nivel de agotamiento y esfuerzo implicados, el tiempo invertido en ocio digital, deportivo, con pares y académico, la concepción sobre la EF y la aptitud para la EF (Tabla 4). Como resultados más destacados, el tiempo invertido en la práctica físico-deportiva mostró una correlación positiva y moderada con el ocio deportivo ( $\mathrm{r}$ = .245), positiva y baja con la aptitud para la EF $(r=.171)$ y negativa y baja con el ocio digital ( $r=-.134)$. El nivel de esfuerzo implicado en la realización de actividades deportivas se relacionó negativa y moderadamente con el ocio deportivo $(\mathrm{r}=-.258)$ y la aptitud para la EF $(\mathrm{r}=$ .344), mientras que reveló una correlación baja con la concepción sobre la EF ( $\mathrm{r}=$-.217). Finalmente, el ocio deportivo reveló una correlación negativa y moderada con el ocio digital $(r=-.324)$, negativa y baja con el ocio académico ( $\mathrm{r}=$-.139), así como un nivel bajo de correlación con la concepción sobre la EF $(r=.112)$ y la aptitud para la EF $(r=.214)$.

\section{Discusión}

Dada la relevancia de la práctica físico-deportiva en la promoción de hábitos saludables en edades tempranas, este estudio pretende concretar cuáles son los deportes más practicados y el modo de hacerlo en una muestra de escolares, así como definir las relaciones existentes entre el nivel de práctica físico-deportiva, el nivel de agotamiento y esfuerzo implicado, el tipo de ocio y las percepciones existentes sobre la EF. En este sentido, algunos estudios de línea similar son los realizados por Barkoukis et al. (2010), Espejo et al. (2015), González-Cutre, Sicilia y Moreno (2011), Martínez et al. (2012) o Moreno-Murcia et al. (2012).

El presente estudio revela que los deportes colectivos de contacto y los individuales sin contacto son los más practicados, con especial protagonismo del fútbol, los deportes acuáticos, el baloncesto y los deportes de raqueta. El modo de práctica más común era el federado, seguido de las actividades extraescolares y el deporte lúdico con pares.
Chillón, Delgado, Tercedor y González-Gross (2002) obtuvieron en su estudio el fútbol, la natación y el tenis como deportes más populares entre niños y niñas. No obstante, también obtuvieron elevadas porcentualidades en ciclismo, contrastando con su escasa práctica en el presente estudio; principalmente debido a diferencias sociodemográficas, dado que la provincia de A Coruña, al ser una zona costera, oferta multitud de actividades en el medio acuático. Por otro lado, MorenoMurcia et al. (2012) obtienen entre los motivos más comunes para la práctica deportiva la salud y el desarrollo de la habilidad, quedando el componente lúdico relegado a un segundo plano. No obstante, estas diferencias son atribuibles a las características de la muestra, pues Moreno-Murcia et al. (2012) realizan su estudio en adultos jóvenes para los que el cuidado de la salud y la imagen corporal tiene una mayor relevancia, mientras que para los niños y niñas en edad escolar tiene mayor importancia motivaciones integradas como es el componente lúdico (García-Calvo et al., 2011; Isorna et al., 2014).

Se pudo observar que el nivel de práctica físico-deportiva, cuantificado en horas semanales, se relacionó positivamente con el ocio deportivo y la aptitud para la EF, y negativamente con el ocio digital. En este sentido, se revela que los escolares que más deporte practican siguen un estilo de vida más saludable, ya que disminuyen los hábitos sedentarios que se relacionan con el ocio digital de pantalla, tal y como muestran Chacón et al. (2015) y Saunders, Chaput y Tremblay (2014) en sus estudios. Asimismo, los niños y niñas físicamente activos se perciben más aptos para la Educación Física, lo que demuestra la importancia de promover un estilo de vida activo desde edades tempranas, ya que tal y como establecen Arriscado et al. (2015) y Haerens, Kirk, Cardon y De Bourdeaudhuij (2011), les permitirá valorar el componente saludable de lamisma.

Los escolares que menos tiempo dedicaban al ocio deportivo y que tenían una peor concepción de la asignatura de EF eran los que mayor nivel de esfuerzo dedicaban en la ejecución de tareas deportivas. Estas premisas se adhieren a connotaciones fisiológicas y cognitivas, ya que un nivel de esfuerzo más elevado ante una actividad de intensidad determinada se asociará a una peor condición física (Muros, CofreBolados, Salvador-Pérez, Castro-Sánchez, Valdivia-Moral y PérezCortés, 2016) y a menores niveles de motivación (Méndez-Giménez et al., 2013). De hecho, Vink, Raudsepp y Kais (2015) muestran en su estudio como aquellos adolescentes que obtienen mayores puntuaciones en motivaciones autodeterminadas son los más físicamente activos. Estas premisas podrían justificar, desde el punto de vista motivacional, por qué los escolares que menos tiempo dedican a la práctica deportiva dedican mayor esfuerzo en la realización de tareas deportivas.

Se reveló que el ocio deportivo mostraba una relación negativa con el ocio digital y el ocio académico, así como una relación positiva con la concepción sobre la EF y la aptitud para la EF. Tal y como se viene estableciendo, resulta evidente que los escolares que mayor tiempo invierten en práctica de AF y deporte sean los menos sedentarios y los que se perciban con mayor aptitud para la EF, tal y como avalan los trabajos de Méndez-Giménez et al. (2013) o Moreno, Cervelló y Moreno (2008). Del mismo modo, la relación negativa entre ocio deportivo y académico-lectura, juegos de intelecto, etc.-, puede venir determinada por una dedicación excesiva a las tareas deportivas, reduciendo considerablemente el tiempo que los escolares dedican a actividades académicas que pueden resultar menos atractivas y lúdicas para este sector (Navarro-Patón et al., 2016; Ros, 2009). Sin embargo, resulta importante señalar como ambas actividades de ocio pueden mostrar una relación positiva, pues Owen et al. (2013) o Staiano y Calvert (2011) señalan como las actividades de intelecto generan beneficios a nivel cognitivo en la práctica deportiva, mejorando la memoria y la capacidad de atención, el desarrollo de las funciones ejecutivas o las habilidades viso-espaciales, etc.

Resulta de interés destacar algunas de las principales limitaciones que presenta este estudio. En primer lugar, cabe destacar aquellas que se adhieren a un estudio de corte transversal, pues aunque permite observar el estado de una cuestión, no pueden desarrollarse relaciones de causa-efecto tal y como permitiría un estudio cuasi-experimental de 
carácter longitudinal. También destacar los instrumentos utilizados, los cuales fueron elaborados explícitamente para realizar esta investigación. En este sentido, hubiese resultado de interés emplear instrumentos previamente validados, los cuales otorgarían mayor potencia estadística a los resultados. Finalmente, destacar los defectos derivados de la muestra de estudio, la cual se compone exclusivamente de alumnado de tercer ciclo de Educación Primaria; siendo interesante ampliarla en investigaciones futuras mediante estudiantes de otras etapas educativas.

En relación a lo expuesto, este estudio alcanza como principales conclusiones:

- Uno de cada diez escolares no practica ningún deporte, mientras que los deportes colectivos con contacto y los individuales sin contacto son los más practicados, especialmente de forma federada, como actividad extraescolar o lúdica con los pares. El fútbol y los deportes acuáticos fueron los deportes más populares, mientras que el balonmano y el atletismo eran los menos practicados.

- El tiempo invertido en la práctica físico-deportiva se relaciona de forma positiva con el ocio deportivo y la aptitud para la EF, y de forma negativa con el ocio digital. El nivel de esfuerzo implicado en el deporte correlaciona negativamente con el ocio deportivo, la concepción sobre la EF y la aptitud para la EF. Finalmente, el ocio deportivo reveló una relación negativa con el ocio digital y el ocio académico, así como una relación positiva con la concepción sobre la EF y la aptitud para la $\mathrm{AF}$.

\section{Referencias}

Alonso, F. J., Carranza, M. D., Rueda, J. D. y Naranjo, J. (2014). Composición corporal en escolares de primaria y su relación con el hábito nutricional y la práctica reglada de actividad deportiva. Revista Andaluza de Medicina del Deporte, 7(4), 137-142. doi: 10.1016/j.ramd.2014.08.001.

Anderson, C. A., Shibuya, A., Thori, N., Swing, E. L., Bushman, B. J., Sakamoto, A., ... y Saleem, M. (2010). Violent video game effects on aggression empathy, and prosocial behaviour in eastern and western countries: a meta-analytic review. Psychological Bulletin, 136(2), 151-173. doi: 10.1037/a0018251.

Arriscado, D., Muros, J. J., Zabala, M. y Dalmau, J. M. (2015). Hábitos de práctica física en escolares: factores influyentes y relaciones con la condición física. Nutrición Hospitalaria, 31(3), 1232-1239. doi: 10.3305\%2Fnh.2015.31.3.8186.

Baena, A., Flores, G. y Barbero, G. (2007). La práctica de actividades físicas y deportivas en el entorno del municipio y su implicación en el currículo de la educación física escolar. Retos. Nuevas tendencias en Educación Física, Deportey Recreación, 11, 54-59.

Barkoukis, V., Ntoumanis, N. y Thogersen, C. (2010). Developmental changes in achievement motivation and affect in physical education: Growth trajectories and demographic differences. Psychology of Sport and Exercise, 11(2), 83-90 doi: 10.1016/j.psychsport.2009.04.008.

Chacón-Cuberos, R., Castro-Sánchez, M., Muros-Molina, J. J., Espejo-Garcés, T. Zurita-Ortega, F. y Linares-Manrique, M. (2016). Adhesión a la dieta mediterránea en estudiantes universitarios y su relación con los hábitos de ocio digital. Nutrición Hospitalaria, 33(2), 405-410. doi: 10.20960/nh.124.

Chacón, R., Espejo, T., Cabrera, A., Castro, M., López, J. F. y Zurita, F. (2015). Exergames para la mejora de la salud en niños y niñas en edad escolar: estudio a partir de hábitos sedentarios e índices de obesidad. RELATEC,14(2), 39-50. doi: 10.17398/1695-288X.14.2.39

Chillón, P., Delgado, M., Tercedor, P. y González-Gross, M. (2002). Actividad físico-deportiva en escolares adolescentes. Retos. Nuevastendencias en Educación Física, Deporte y Recreación, 1, 5-12.

Dias, S. y Gama, A. (2016). Health promotion teaching in post-graduation in international public health in the context of the Bologna process. Saude e Sociedade, 25(3), 771-785.

Espejo, T., Cabrera, A., Castro, M., López, J. F., Zurita, F. y Chacón, R. (2015). Modificaciones de la obesidad a través de la implementación de herramientas físico-posturales. Retos. Nuevas tendencias en Educación Física, Deportey Recreación, 28,78-83.

Estruch, R., Ros, E., Salas-Salvadó. J., Covas, M. I., Corella, D., Arós, F., ... y Lamuela-Raventos, R. M. (2013). Primary prevention of cardiovascular disease with a Mediterranean diet. New England Journal of Medicine, 368(14), 127990. doi: 10.1056/NEJMoa1200303.

García-Calvo, T., Sánchez, P. A., Leo, F. M., Sánchez, D. y Amado, D. (2011). Incidencia dela Teoría de la Autodeterminación sobre la persistencia deportiva. RICYDE, 7(25), 267-276.
Gómez-Mármol, A. y De la Cruz-Sánchez, E. (2013). Diferencias de género y de nivel académico en la utilidad percibida de la educación física escolar. Journal of Sport and Health Research, 5(2), 193-202.

González-Cutre, D., Sicilia, A. y Moreno, J.A. (2011). Un estudio cuasi-experimental de los efectos del clima motivador tarea en las clases de Educación Física. Revista de Educación, 356, 677-700. doi: 10-443811988-592X-RE-2010356-056.

Haerens, L., Kirk, D., Cardon, G. y De Bourdeaudhuij, I. (2011). Toward the development of apedagogical model for health-based physical education. Quest, 63(3), 321-338. doi: 10.1080/00336297.2011.10483684.

Isorna, M., Rial, A. y Vaquero-Cristóbal, R. (2014). Motivaciones para la práctica deportiva en escolares federados y no federados. Retos. Nuevas tendencias en Educación Física, Deporte y Recreación, 11, 80-84.

Kane, J.E.(2015). Psychological aspects of Physical Edication and Sport. Routledge: New York.

Martínez, J., Contreras, O., Aznar, S. y Lera, A. (2012). Niveles de actividad física medido con acelerómetro en alumnos de $3^{\circ}$ ciclo de Educación Primaria: actividad física diaria y sesiones de Educación Física. Revista de Psicología del Deporte, 21(1), 117-123.

Méndez-Giménez, A., Fernández-Río, J., Cecchini, J. A. y González, C. (2013). Perfiles motivacionales y sus consecuencias en educación física. Un estudio complementario de metas de logro 2x2 y autodeterminación. Revista de Psicología del Deporte, 22(1), 29-38.

Moreno, J.A., Cervelló, E. y Moreno, R. (2008). Importancia de la práctica físicodeportiva y del género en el autoconcepto físico de los 9 a los 23 años. International Journal of Clinical and Health Psychology, 8(1), 171-183.

Moreno-Murcia, J. A., Borges, F., Marcos, P. J., Sierra, A. C. y Huéscar, E. (2012). Motivación, frecuencia y tipo de actividad en practicantes de ejercicio físico. Revista Internacional de Medicina y Ciencias de la Actividad Física y del Deporte, 12(48), 649-662.

Moreno-Murcia, J.A., Zomeño, T., Marín, L. M. y Cervelló, E. (2013). Percepción de la utilidad e importancia de la educación física según la motivación generada por el docente. Revista de Educación, 362, 380-401.

Muros, J. J., Cofre-Bolados, C., Salvador-Pérez, S., Castro-Sánchez, M., ValdiviaMoral, P. y Pérez-Cortés, A. J. (2016). Relación entre nivel de actividad física y composición corporal en escolares de Santiago (Chile). Journal of Sport and Health Research, 8(1), 83-92.

Navarro-Patón, R., Rodríguez, J.E. y Eirín, R. (2016). Análisis de la satisfacción de las necesidades psicológicas básicas, motivación y disfrute en Educación Física en Primaria. Sportis. Scientific Journal of School Sport, Physical Education and Psychomotricity, 2(3), 439-455. doi: 10.17979/sportis.2016.2.3.1758.

OMS (2015). Estrategia mundial sobre régimen alimentario, actividad física y salud. Sobrepeso y obesidad infantil. Recuperado el día 5 de noviembre de 2015, desde: http://www.who.int/dietphysicalactivity/childhood/es/

Owen, K. B., Astell-Burt, T. y Lonsdale, C. (2013). The relationship between selfdetermined motivation and physical activity in adolescent boys. Journal of Adolescent Health, 53(3), 420-422. doi: 10.1016/j.adohealth.2013.05.007.

Reverter, J., Plaza, D., Jové, M. C. y Hernández, V. (2014). Actividad físicodeportiva extraescolar en alumnos de primaria: el caso de Terrevieja (Alicante). Retos, 25, 48-52.

Rodríguez, G (2016).Actitudes y hábitos conductuales hacia la práctica deportiva en el medio rural en función del género. Journal of Sport and Health Research, $8(2), 115-128$.

Ros, I. (2009). La implicación del estudiante con la escuela. Revista de Psicodidáctica, 14(1), 79-92. doi: 10.1387/RevPsicodidact.252.

Saunders, T. J., Chaput, J. P. y Tremblay, M. S. (2014). Sedentary behaviour as an emerging risk factor for cardiometabolic diseases in children and youth. Canadian Journal of Diabetes, 38(1), 53-61. doi: 10.1016/j.jcjd.2013.08.266.

Sicilia,A., Águila, C., González-Cutre, D. y Moreno-Murcia, J.A. (2011). Factores motivacionales y experiencia autotélica en el ejercicio físico: propuesta de un modelo explicativo. Universitas Psychologica, 10(1), 125-135.

Staiano, A. E. y Calvert, S. L. (2011). Exergames for physical education courses: Physical, social, and cognitive benefits. Child Development Perspectives, 5(2), 93-98. doi: 10.1111/j.1750-8606.2011.00162.x.

Van Rooij,A. J., Schoenmakers, T. M., Vermulst,A., Vermulst,A., Van den Eijden, R. y Van de Mheen, D. (2010). Online video game addiction: identification of addicted adolescent gamers. Addiction, 106(1), 205-210. doi: 10.1111/j.13600443.2010.03104.x.

Vink, K., Raudsepp, L. y Kais, K. (2015). Intrinsic motivation and individual deliberate practice are reciprocally related:Evidence from a longitudinal study of adolescent team sport athletes. Psychology of Sport and Exercise, 16(3), 1-6. doi: 10.1016/j.psychsport.2014.08.012. 